\title{
Prospects for neutrino-flavor physics with in-ice radio detectors
}

\author{
Christian Glaser, ${ }^{a, *}$ D. García-Fernández ${ }^{b, c}$ and Anna Nelles ${ }^{b, c}$ \\ ${ }^{a}$ Uppsala University, Box 516, S-75120 Uppsala, Sweden \\ ${ }^{b}$ DESY, Platanenallee 6, 15738, Zeuthen, Germany \\ ${ }^{c}$ ECAP, Friedrich-Alexander Universität Erlangen-Nürnberg, Erwin-Rommel-Straße 1, 91058 Erlangen, \\ Germany \\ E-mail: christian.glaser@physics.uu.se
}

The detection of the radio emission following a neutrino interaction in ice is a promising technique to obtain significant sensitivities to neutrinos with energies above $10 \mathrm{PeV}$. The detectable radio emission stems from particle showers in the ice. So far, detector simulations have considered only the radio emission from the primary interaction of the neutrino. We present how the simulation code NuRadioMC was extended to cover secondary interactions from muons and taus. Muons and taus, created by an interaction of the corresponding neutrino, can create several additional detectable showers during their propagation through the ice, which adds up to $25 \%$ to the effective volume of neutrino detectors. It provides a signature for the neutrino flavor and improves event reconstruction if multiple of these showers are detected. We simulated the signatures of secondary interactions for the RNO-G detector in Greenland and the proposed radio detector of IceCubeGen2. We also find that the background of atmospheric muons from cosmic rays is non-negligible for in-ice arrays and that an air shower veto should be considered helpful for radio detectors.

$37^{\text {th }}$ International Cosmic Ray Conference (ICRC 2021)

July 12th-23rd, 2021

Online - Berlin, Germany

\footnotetext{
${ }^{*}$ Presenter
} 


\section{Introduction}

The radio technique offers a cost efficient way to build a detector with sensitivity to ultra-highenergy neutrinos with energies beyond $30 \mathrm{PeV}$. An array of autonomous radio detector stations, each consisting of multiple antenna receivers, allows to instrument huge volumes of ice to search for radio signals generated by neutrino interactions in the ice via the Askaryan effect. The technology has been developed in small pilot arrays over the last decade and the hardware has proven to work reliably in harsh polar conditions [1,2]. Currently the first discovery scale detector, the Radio Neutrino Observatory in Greenland (RNO-G) [3] is under construction. A detector with similar sensitivity that builds up on the ARIANNA pilot array is proposed for the Ross Ice Shelf [4] and an order of magnitude larger array is foreseen as part of IceCube-Gen2 [5].

With experimental efforts reaching maturity, the software and simulation tools have to follow along. Up until recently, simulation tools focused on calculating effective volumes for different types of neutrino detectors, thereby including only the primary neutrino interaction. The nominal radio signal originates from the particle cascade initiated by the neutrino interaction in the ice. For neutral current (NC) interactions, a so-called hadronic shower is initiated by the breakup of the parton the neutrino interacted with. For charged current (CC) interactions of electron neutrinos, the generated electron also induces an electromagnetic particle shower. The muon and tau leptons that are generated in $\mathrm{CC}$ interactions of the respective neutrinos were mostly ignored in systematic detector simulations which changed through the work first presented in [6], where a more detailed discussion of the signatures of secondary leptons in radio-neutrino detectors in ice are discussed. These proceedings summarize the findings, report on technical improvements and cross-checks made since, and present a new simulation for a detector at the South Pole.

The NuRadioMC simulation code was developed to enable a flexible simulation of neutrino detectors and the optimization of their layouts in preparation for larger scale deployments [7]. We have extended NuRadioMC to study contributions of interactions from secondary leptons, which is presented here. This code provides the first calculation of radio emission from neutrino interaction to a detector in ice containing in a systematic way all the possible showers produced by secondary leptons. This work also allows us to study a potential background of atmospheric muons, i.e., high-energy muons generated in cosmic-ray induced air showers, that generate radio signals when propagating through the ice.

We use the lepton propagation code PROPOSAL [8] to propagate muon and tau leptons through ice and write out all energy deposits above $10^{14} \mathrm{eV}$. In Fig. 1, an example of a $10^{18} \mathrm{eV}$ muon neutrino propagating through ice is shown. The muon induces several particle cascades with energies close to or above $10^{17} \mathrm{eV}$ which produces a strong radio signal similar in strength to the radio signal from the initial neutrino interaction. The smaller the deposited energy, the more often these energy deposits occur. Several close-by showers can interfere constructively, potentially increasing the shower energy to the sum of shower energies of the individual showers. This suggests that interference between showers should be taken into account when calculating the expected radio emission. In these proceedings, we present how NuRadioMC was modified to take into account interference between nearby showers which is an extension to our previous work where all showers were treated independently [6]. 


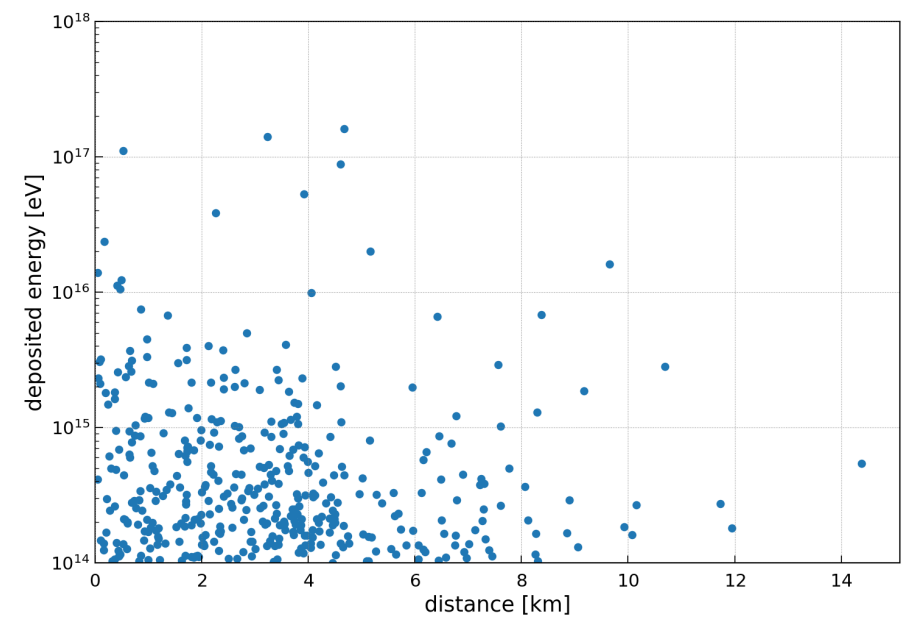

Figure 1: Stochastic energy losses above $10^{14} \mathrm{eV}$ of a $10^{18} \mathrm{eV}$ muon propagating through the ice. Each energy deposit initiates a particle cascade that could produce detectable Askaryan radio emission.

\section{Integration into NuRadioMC}

To accommodate the simulation of several in-ice showers (that generate Askaryan radiation) we adapted the internal structure of NuRadioMC (see Fig. 2). All showers originating from the same initial neutrino interaction are grouped into an event group. For neutral current (NC) interactions of neutrinos, the event group just consists out of a single hadronic shower. For an electron neutrino charged current (CC) interaction, an event group comprises a hadronic shower and an additional electromagnetic shower (induced by the generated electron). For a muon or tau neutrino CC interaction, an event group consists of the initial hadronic shower (induced by the initial neutrino interaction) plus an arbitrary amount of secondary showers that are induced by the propagation of the muon or tau through the ice (cf. Fig 1).

NuRadioMC is designed to reproduce the behavior of a radio detector as precisely as possible. As typically each radio detector station operates as an autonomous unit, NuRadioMC also treats each detector station independently. Hence, NuRadioMC first loops over all detector stations. Then, each shower is considered. For each shower every readout channel (the different antennas of a station) is considered, and for every channel all possible signal trajectories are calculated. Due to the changing index of refraction in the upper $O(100 \mathrm{~m})$ of an ice sheet, one typically gets two trajectories, one direct path to the antenna, and a second one from a reflection at the surface or refraction back down. For sites with a reflective bottom layer, such as the Ross ice shelf, even more signal trajectories are possible.

For every shower, channel and signal trajectory, NuRadioMC calculates the Askaryan signal that arrives at the antenna and performs a full detector simulation taking into account the detector response as well as time delays due to cables etc. This results in a voltage time series that the readout system would see for this combination of shower, channel and signal trajectory. Then, all voltage traces are ordered in time and grouped into events with similar arrival times. The grouping is based on the minimum separation between two succeeding signals and is typically set to $1 \mu \mathrm{s}$ but depends on the details of the radio detector hardware. 


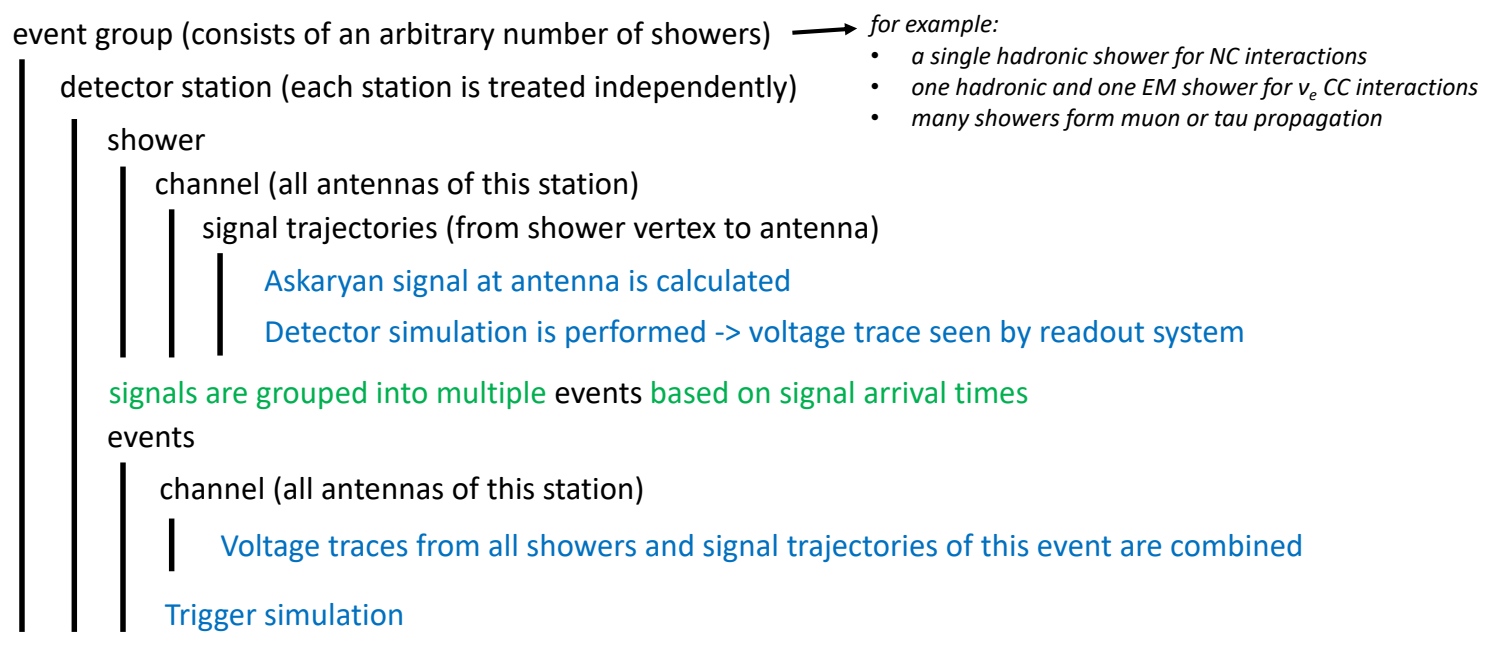

Figure 2: Flow chart of the internal looping structure of NuRadioMC. See text for details.

For every event and for every channel, NuRadioMC combines the signals from the showers and trajectories that belong to this event and channel. This provides an accurate prediction of the signals that would be measured by a radio detector and takes into account possible interference between the signals from different showers and/or signal trajectories. Finally, the trigger is simulated to determine if an event will be recorded or not.

This structure enables an accurate simulation of the following scenarios: First, most naturally, it enables the simulation of many showers from muon or tau interactions including potential interference of nearby showers. Second, it enables the simulation of a single shower that has two (or more) signal trajectories to a receiver with significant differences in propagation time. The new structure will create two (or more) events for this shower, just like a radio detector would trigger independently on these time delayed signals. Third, the new structure enables the simulation of new physics scenarios and/or new emission models. For example, the LPM effect can be modelled manually by placing many low-energy electromagnetic showers at different locations. A user would only need to write a custom event generator that defines the shower positions, times, energies and directions. With this information, NuRadioMC will predict the observable radio signals.

\subsection{Impact of interference}

In Fig. 3, the effect of including interference in the simulation is shown. We simulated a flux of atmospheric muons which was propagated through the ice using the PROPOSAL code (cf. Fig. 1). We further simulated a radio detector station at the South Pole with a phased array trigger at $100 \mathrm{~m}$ depth that was approximated by a single dipole antenna at a trigger threshold of 1.5 times the noise RMS in the frequency band from $100 \mathrm{MHz}$ to $730 \mathrm{MHz}$ and a noise temperature of $250 \mathrm{~K}$. We performed the simulation once taking into account interference between showers (as described above) and a second time where each shower was treated individually (as done in our previous work [6]). We simulated exactly the same events which allows us to compare the results on an event-by-event basis. We find that the majority of events is triggered in both cases. An additional $1 \%$ is only triggered if each shower is simulated individually which suggests that some showers 


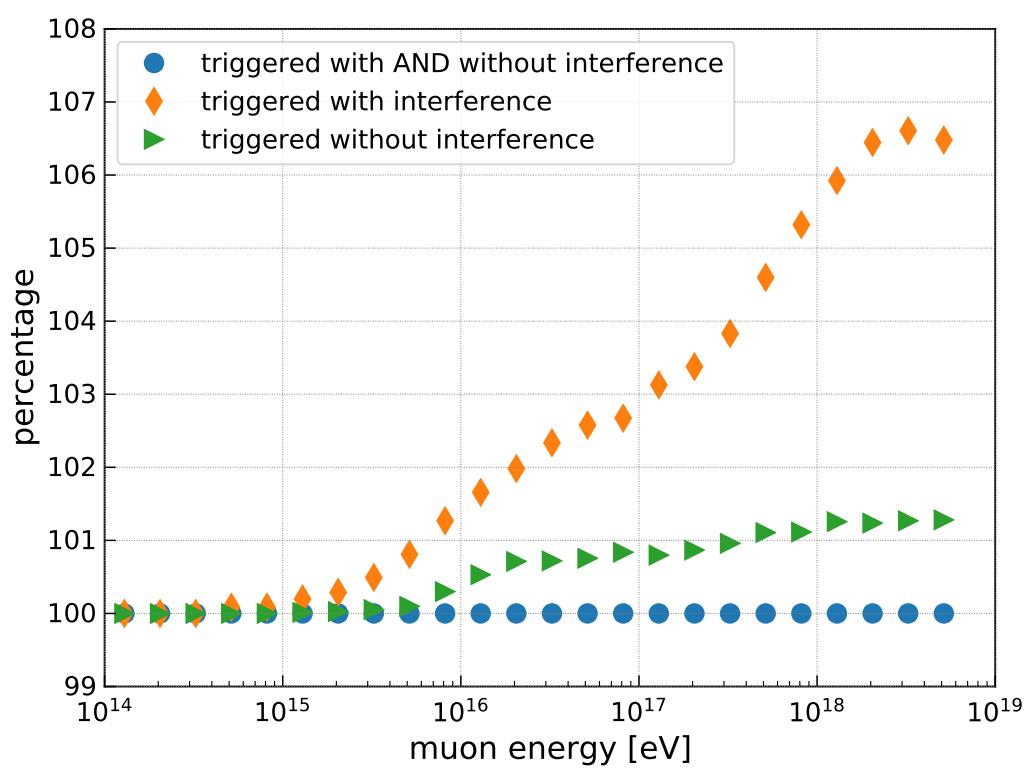

Figure 3: Impact of interference between showers. See text for details.

also interfere destructively. If we take into account interference, up to $7 \%$ additional events are observed.

If we just look at the number of triggered events, the effect of interference is at a few percent level, thus, the approximation done in our previous work was justified to estimate event numbers. However, more importantly, even though an event is triggered with and without interference, interference will modify the pulse shapes which will be important to interpret experimental data. Thus, the improved treatment should be used for signal simulations.

\section{Muons and Taus from neutrino interactions}

We have also investigated the signals of the secondary showers produced by muons and taus after a CC neutrino interaction has taken place in the ice. In our earlier work [6] we have simulated a generic detector consisting of a $10 \times 10$ square array of $100 \mathrm{~m}$ deep dipoles with $1.5 \sigma$ threshold at the RNO-G site in Greenland. Here, we present a similar setup of an array of $11 \times 13$ stations on a square grid with $2 \mathrm{~km}$ spacing and a phased array trigger at $200 \mathrm{~m}$ depth at the South Pole which is the site foreseen for IceCube-Gen2 [5]. We simulate an isotropic flux of muon and tau neutrinos, calculate the radio emission from the initial and secondary interactions, and determine which signals fulfill the trigger condition.

As a first step, we are particularly interested in the increase of observable events due to the secondary interactions. This is typically quantified using the effective volume which is the sum of triggered events divided by the total number of simulated neutrinos multiplied by the simulation volume where each neutrino is weighted with the probability of reaching the simulation volume. The results are presented in Fig. 4 for muon neutrinos (left) and tau neutrinos (right). In the case of muon neutrinos, we find that the effective volume is increased by more than $40 \%$ at neutrino energies around $3 \times 10^{18} \mathrm{eV}$. At higher energies, the number of observable secondary interactions increases 

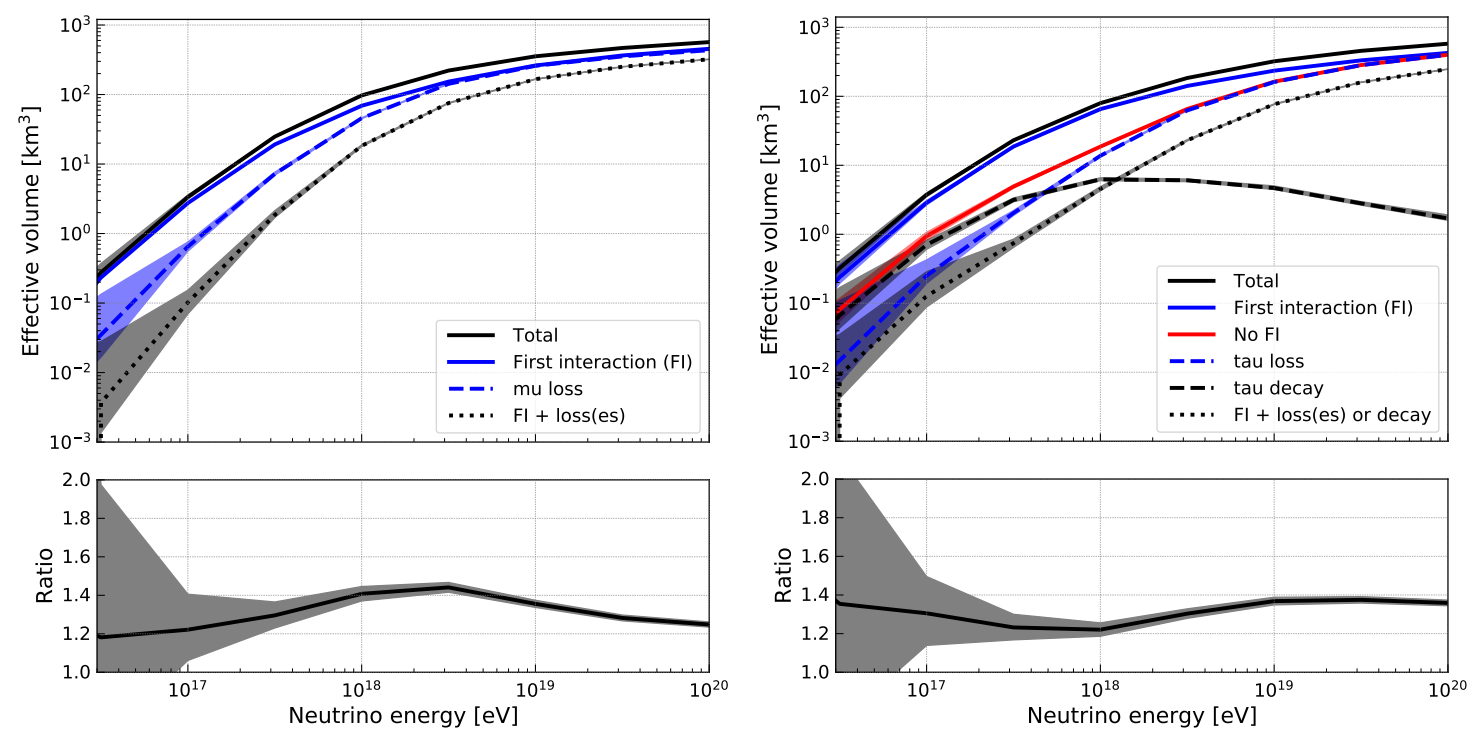

Figure 4: Left: Muon neutrinos, right: tau neutrinos. Top panel: muon/tau neutrino effective volumes for a $11 \times 13$ array at the South Pole with a $2 \mathrm{~km}$ spacing and a 4 channel phased-array trigger system at $200 \mathrm{~m}$. The bands represent the uncertainty assuming a Poisson distribution. There are different types of effective volume depicted in the figure. The events that have been triggered at least by the shower induced by the first neutrino interaction are represented by the 'First interaction' (FI) curve, which corresponds to what was previously obtained without considering secondary interactions. This work adds the following curves: 'No FI': Events not triggered by the first neutrino interactions but by secondary interactions. 'tau decay': events triggered by the products of the tau decay. 'tau loss': triggered by the tau stochastic losses during propagation. 'mu loss': triggered by the muon stochastic losses during propagation. 'FI+losses': events triggered by the neutrino first interaction and at least a stochastic energy loss. 'FI + Loss(es) or decay': events triggered by the neutrino first interaction and by the tau decay or one or several tau stochastic losses. 'Total' the total effective volume. Bottom panel: ratio of the total effective volume over the first interaction effective volume.

further and almost equals the number of observable first interactions. However, for an increasing fraction of events, the array will observe the signal from the first and a secondary interaction simultaneously in multiple stations. While this limits the increase in total effective volume, it will provide very interesting event signatures to study flavor physics at $\mathrm{EeV}$ energies.

The situation for tau neutrino CC interactions is similar but more complex because of the additional decay mode. At energies up to $3 \times 10^{17} \mathrm{eV}$, an observable secondary interaction is most likely due to the tau decay. At these energies, the tau decay length is around $1 \mathrm{~km} \mathrm{[7]} \mathrm{and} \mathrm{the} \mathrm{tau} \mathrm{is}$ likely to still decay in the ice sheet even for more vertical directions. At a tau energy of $10^{18} \mathrm{eV}$, the average decay length is already $20 \mathrm{~km}$ which makes it unlikely that the tau decays in the ice sheet. Instead, stochastic energy losses during the tau propagation become dominant at energies beyond $3 \times 10^{17} \mathrm{eV}$. The increase in effective volume increases from $20 \%$ at $10^{18} \mathrm{eV}$ to $40 \%$ at $10^{19} \mathrm{eV}$. At the highest studied energy of $10^{20} \mathrm{eV}$, measuring the first interaction is equally likely as measuring one of the secondary interactions. As for the muon case, at higher energies the fraction of events where the first and one of the secondary interactions is observed increases.

The simultaneous observation of the first and one of the secondary interactions provide an interesting event signature. We show one example in Fig. 5 where the initial tau neutrino interaction and a secondary interaction is observed in three detector stations. Through this channel, we 


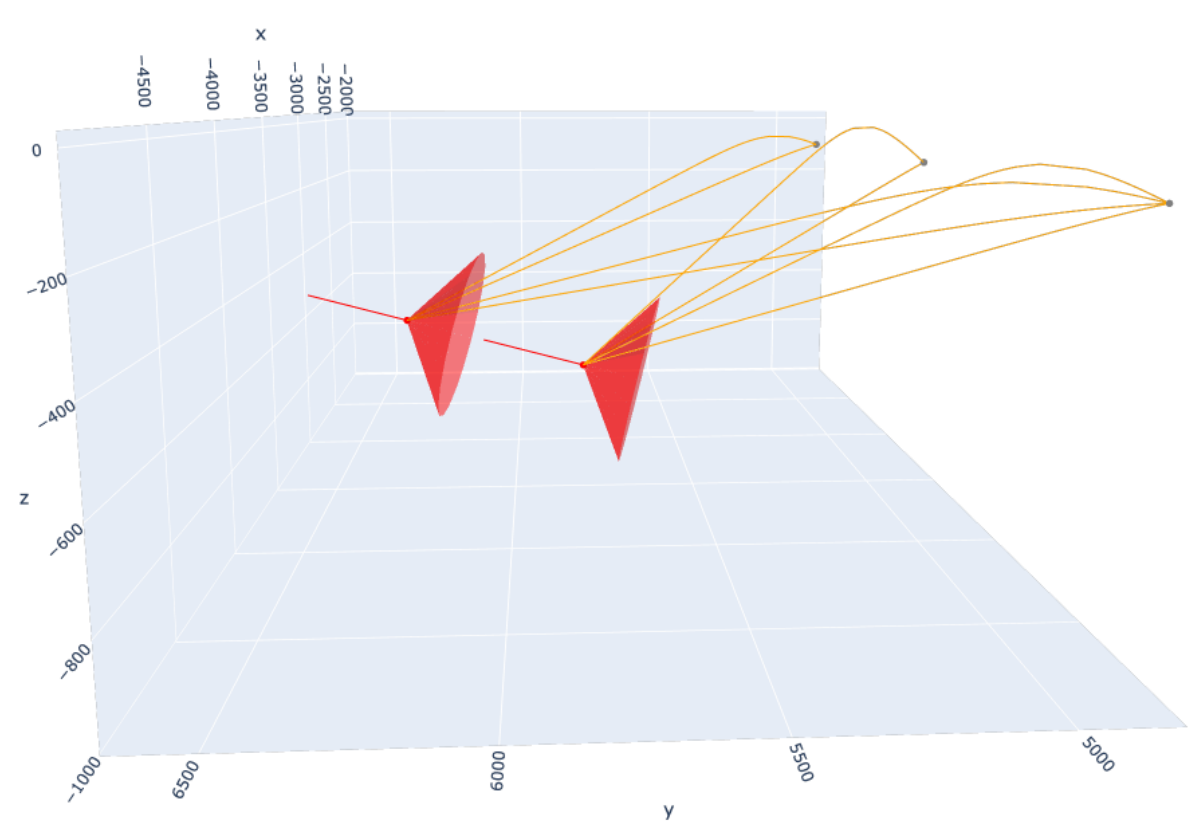

Figure 5: Three dimensional plot of a double-cascade event. The first shower is caused by a $1.84 \mathrm{EeV}$ neutrino. The resulting tau travels for several hundred meters and creates a hadronic shower via photonuclear interaction. The red line represents the particles trajectories, while the red cones indicate the Cherenkov cones for both showers. The three triggered stations are represented by grey dots, and the yellow lines are the paths followed by the waves (direct and refracted) that arrive at the stations. The axes units are meters. Figure and caption adapted from [6].

envision obtaining flavor sensitivity and distinguishing muon and tau neutrino $\mathrm{CC}$ interactions from the rest. Apart from estimating the flavor, these events also provide an improved event reconstruction capability. In particular the neutrino direction can be estimated more precisely due to the large lever arm between the first and secondary interaction vertex.

\section{Conclusions}

We presented detailed calculations of the signatures of secondary leptons in radio detectors for high-energy neutrinos. Muon and tau leptons created in CC interactions of the respective neutrinos generate Askaryan emission dominantly due to stochastic energy losses during propagation. Including these interactions in the simulation increases the predicted sensitivity of a radio neutrino detector by up to $40 \%$ for both muon and tau neutrinos. Averaged over all flavors and assuming a 1:1:1 flavor distribution at the detector, secondary interactions add up to $25 \%$ to the effective volume.

We modified NuRadioMC to simulate interference between nearby showers and found that the 
number of observable events is increased by a few percent if interference is taking into account. This modification turns NuRadioMC into a general toolkit for simulating any physics scenarios that generate (multiple) in-ice showers. Users can write their own event generator that defines shower positions, times, energies, and direction and NuRadioMC will calculate the resulting radio signal in a detector. This can be used for example to study beyond standard model processes and their signatures in a radio detector, or a modeling of the LPM effect by manually defining the spatially displaced sub showers.

The simultaneous observation of the initial neutrino interaction and a secondary interaction from muons or taus provides a way to identify the neutrino flavor and will yield an improved event reconstruction. We note that also electron neutrino charged-current interactions provide flavor sensitivity in a radio detector when the generated electromagnetic shower is significantly impacted by the LPM effect [9].

This work also allows us to calculate a potential background of radio signals from high-energy muons propagating through the ice that were created by cosmic-ray interactions in the atmosphere. Models for the high-energy muon flux exhibit large uncertainties as no experimental data exists at the relevant energies. Using one current model of the cosmic-ray flux, its composition, and hadronic interaction models, non-negligible event rates of $O(1)$ event per year are expected for an array of the size of IceCube-Gen2 [5]. Given the uncertainties in these predictions an air shower veto should be considered helpful for radio detectors.

\section{Acknowledgements}

The authors would like to thank Dan Smith (UChicago) and Brian Clark (MSU) for running part of the simulations for the South Pole site. We would like to thank our colleagues from the radio neutrino community for the lively discussions. The authors acknowledge funding from the German Research Foundation (DFG) under grant NE 2031/2-1 (DGF, AN) and GL 914/1-1 (CG). We thank the Swedish National Infrastructure for Computing (SNIC) at Uppsala Multidisciplinary Center for Advanced Computational Science (UPPMAX) for providing computing resources for this work.

\section{References}

[1] ARIANNA Collaboration, A. Anker et al. JCAP 03 (2020) 053.

[2] ARA Collaboration, P. Allison et al. Phys. Rev. D 102 (2020) 043021.

[3] RNO-G Collaboration, J. A. Aguilar et al. JINST 16 P03025 2021.

[4] S. Barwick for the ARIANNA collaboration PoS ICRC2021 (these proceedings) 1067.

[5] S. Hallmann for the IceCube-Gen2 collaboration PoS ICRC2021 (these proceedings) 1183.

[6] D. García-Fernández, C. Glaser, and A. Nelles Phys. Rev. D 102 (2020) 083011.

[7] C. Glaser et al. European Physical Journal C80 (2020) 77.

[8] J.-H. Koehne et al. Computer Physics Communications 184 no. 9, (2013) 2070 - 2090.

[9] S. Stjärnholm, O. Ericsson and C. Glaser PoS ICRC2021 (these proceedings) 1055. 